\title{
Dual Generalized Order Statistics from Gompertz-Verhulst Distribution and Characterization
}

\author{
M. I. Khan * \\ Department of Mathematics, Faculty of Science, Islamic University of Madinah, Madinah, Kingdom of Saudi Arabia
}

\begin{abstract}
The dual generalized order statistics is a unified scheme which contains the well known decreasingly ordered random variables such as (reversed) order statistics, lower record values and lower Pfeifer record values. In this article, characterization results on Gompertz-Verhulst distribution through the conditional expectation of dual generalized order statistics based on non-adjacent dual generalized order statistics are given. These relations are deduced for moments of reversed order statistics, order statistics and lower record values. Further a characterization result through the truncated moment is also derived.
\end{abstract}

Keywords Dual generalized order statistics, reversed order statistics, lower record value, conditional expectations, truncation and characterization.

AMS 2010 subject classifications 62E15, 62E10, 62G30

DOI: $10.19139 /$ soic-2310-5070-652

\section{Introduction}

Models of ordered random variables are important and received a great attention from many researchers during the past century. These models are order statistics, sequential order statistics, record values, Pfeifer record values, progressively censored order statistics and generalized order statistics, see M. Q. Shahbaz et al. [1].

Kamps [2] has proposed generalized order statistics (gos) as a unified models of ordered random variables which produce several models as a special case. The comprehensive model for ordered random variables in ascending order is generalized order statistics. Often it happen that the sample is arranged in descending order for example the life length of an electric bulb arranged from highest to lowest. In such situations the distributional properties of variables cannot be studied by using the models of ordered random variables. The study of distributional properties of such random variables is studied by using the inverse image of gos and is popularly known as dual generalized order statistics. The dual generalized order statistics (dgos) was introduced by Burkschat et al. [3] as a unified model for descendingly ordered random variables like reverse order statistics, lower record values and lower Pfeifer record values.

The characterization of probability distributions is mainly useful in the area of goodness of fit tests, which is helpful in the construction of statistical tests. The book by Kagan et al. [4] provides the reader a good idea of the different types of properties which have been used for the characterization. Further it is a valuable source of information about the whole field of characterization of probability distributions.

Characterization of probability distributions play an important role in probability and statistics. A probability distribution can be characterized by several methods. In recent years there has been a great interest in the

*Correspondence to: M. I. Khan (Email: izhar.stats@gmail.com)

ISSN 2310-5070 (online) ISSN 2311-004X (print)

Copyright (C) 2020 International Academic Press 
characterization of probability distributions through conditional expectations. For example, the development of the general theory of characterizations of probability distributions by conditional expectations began with work of Kotlarski [5], Talwalker [6] and Galambus and Kotz [7] are notable.

Further the development on the characterization of probability distributions by conditional expectations continued with the contributions of many authors among them, Gupta and Ahsanullah [8], Zora et al. [9], Khan et al. [10] and Gupta and Anwar [11] among others.

Several authors have utilized the concept of dual generalized order statistics in characterization of distributions including Ahsanullah [12, 13], Mbah and Ahasanullah [14], Khan et al. [15, 16], Faizan and Khan [17], Tavanagar [18], Khan and Faizan [19], Khan and Khan [20] and Khan and Khan [21].

The dual generalized order statistics (dgos) or sometimes called lower generalized order statistics (lgos) is a combined mechanism of studying random variables arranged in descending order. The technique was introduced by Burkschat et al. [3] and is defined in the following.

Let $F(x)$ be an absolutely continuous distribution function $(d f)$ with the probability density function $(p d f)$ $f(x)$. Further, let $n \in N, n \geq 2, k>0, \tilde{m}=\left(m_{1}, m_{2}, \ldots ., m_{n-1}\right) \in \mathfrak{R}^{n-1}, M_{r}=\sum_{j=r}^{n-1} m_{j}$. Such that $\gamma_{r}=$ $k+n-r+M_{r}>0, \forall r \in\{1,2, \ldots, n-1\}$. Then $X^{\prime}(r, n, \tilde{m}, k), r=1,2, \ldots, n$ are called (dgos) if their joint $p d f$ is given by,

$$
k\left(\prod_{j=1}^{n-1} \gamma_{j}\right)\left(\prod_{i=1}^{n-1}\left[F\left(x_{i}\right)\right]^{m_{i}} f\left(x_{i}\right)\right)\left[F\left(x_{n}\right)\right]^{k-1} f\left(x_{n}\right),
$$

for $F^{-1}(1)>x_{1} \geq x_{2} \geq \ldots \geq x_{n}>F^{-1}(0)$.

Here two cases may be considered:

1.1 Case I: $m_{i}=m_{j}=m, i, j=1,2, \ldots, n-1$.

The probability density function of the $r^{t h}-d g o s$ is given by,

$$
f_{X^{\prime}(r, n, m, k)}(x)=\frac{C_{r-1}}{(r-1) !}[F(x)]^{\gamma_{r}-1} f(x) g_{m}^{r-1}[F(x)],-\infty<x<\infty .
$$

The joint probability density function of the $r^{t h}$ and $s^{t h}-d g o s$ is given by,

$$
\begin{gathered}
f_{X^{\prime}(r, n, m, k), X^{\prime}(s, n, m, k)}(x, y)=\frac{C_{s-1}}{(r-1) !(s-r-1) !}[F(x)]^{m} f(x) g_{m}^{r-1}[F(x)] \\
\times\left[h_{m}(F(y))-h_{m}(F(x))\right]^{s-r-1}[F(y)]^{\gamma_{s}-1} f(y),-\infty<y<x<\infty .
\end{gathered}
$$

The conditional $p d f$ of $X^{\prime}(s, n, m, k)$ given $X^{\prime}(r, n, m, k)=x, 1 \leq r \leq s \leq n$, is

$$
f_{s \mid r}(y \mid x)=\frac{C_{s-1}}{C_{r-1}(s-r-1) !} \frac{\left[(F(x))^{m+1}-(F(y))^{m+1}\right]^{s-r-1}[F(y)]^{\gamma_{s}-1}}{(m+1)^{s-r-1}[F(x)]^{\gamma_{r+1}}} f(y), y<x
$$

where,

$$
\begin{aligned}
& C_{r-1}=\prod_{i=1}^{r} \gamma_{i}, \gamma_{i}=k+(n-i)(m+1), \\
& h_{m}(x)= \begin{cases}-\frac{1}{m+1} x^{m+1} & , m \neq-1 \\
-\log x & , m=-1\end{cases}
\end{aligned}
$$

and

$$
g_{m}(x)=h_{m}(x)-h_{m}(1), x \in[0,1) .
$$

1.2 Case II: $\gamma_{i} \neq \gamma_{j}, i \neq j i, j=1,2, \ldots, n-1$.

The probability density function of the $r^{t h}-d g o s$ is given by,

$$
f_{X,(r, n, \tilde{m}, k)}(x)=C_{r-1} f(x) \sum_{i=1}^{r} a_{i}(r)[F(x)]^{\gamma_{i}-1},-\infty<x<\infty
$$


The joint probability density function of the $r^{t h}$ and $s^{t h}-d g o s$ is given by,

$$
f_{X,(r, n, \tilde{m}, k), \quad X,(s, n, \tilde{m}, k)}(x, y)=C_{s-1} \sum_{i=r+1}^{s} a_{i}^{(r)}(s)\left[\frac{F(y)}{F(x)}\right]^{\gamma_{i}} \sum_{i=1}^{r} a_{i}(r)[F(x)]^{\gamma_{i}} \frac{f(x)}{F(x)} \frac{f(y)}{F(y)}
$$

where

$$
a_{i}(r)=\prod_{j(\neq i)=1}^{r} \frac{1}{\left(\gamma_{i}-\gamma_{j}\right)}, \quad \gamma_{i} \neq \gamma_{j}, \quad 1 \leq i \leq r \leq n
$$

and

$$
a_{i}^{(r)}(s)=\prod_{j(\neq i)=r+1}^{s} \frac{1}{\left(\gamma_{i}-\gamma_{j}\right)}, \quad \gamma_{i} \neq \gamma_{j}, r+1 \leq i \leq s \leq n .
$$

The conditional $p d f$ of $X^{\prime}(s, n, \tilde{m}, k)$ given $X^{\prime}(r, n, \tilde{m}, k)=x, 1 \leq r \leq s \leq n$, is

$$
f_{s \mid r}(x)=\frac{c_{s-1}}{c_{r-1}} \sum_{i=r+1}^{s} a_{i}^{(r)}(s)\left[\frac{F(y)}{F(x)}\right]^{\gamma_{i}-1} \frac{f(y)}{F(x)}, x>y .
$$

If $m=0, k=1$, then $X^{\prime}(r, n, m, k)$ reduces to $(n-r+1)^{t h}$ order statistic $X_{n-r+1: n}$ from the sample $X_{1}, X_{2}, \cdots, X_{n}$ and when $m \rightarrow-1$ then $X^{\prime}(r, n, m, k)$ reduces to $r^{t h}$ lower $k$ record values.

\subsection{Gompertz-Verhulst distribution}

A continuous random variable $X$ is said to have Gompertz-Verhulst distribution if its distribution function $(d f) F(x)$ and probability density function $(p d f) f(x)$ are given, respectively,

$$
\begin{gathered}
F(x)=\left[1-\rho e^{-\lambda x}\right]^{\alpha}, \beta \leq x<\infty . \\
f(x)=\alpha \lambda \rho e^{-\lambda x}\left(1-\rho e^{-\lambda x}\right)^{\alpha-1}, \beta \leq x<\infty .
\end{gathered}
$$

where $\beta=\frac{\ln (\rho)}{\lambda}$ and $\rho, \lambda$ and $\alpha \geq 0$. The above model was used by Gompertz and Verhulst known to compare human morality tables and to represent populations growth, see Gompertz [22] and Verhulst [23, 24, 25].

The $p d f$ of Gompertz - Verhulst distribution can take different shapes. It is unimodal for $\alpha>1$ and reversed ' $\mathrm{J}$ ' shaped for $\alpha \leq 1$. Further, the density function of Gompertz- Verhulst distribution is log-convex if $\alpha \leq 1$ and log-concave if $\alpha \geq 1$. It has an increasing hazard function if $\alpha>1$ and decreasing hazard function if $\alpha<1$ and for $\alpha=1$ the hazard function is constant. Putting $\rho=1$ in (10) Gompertz -Verhulst distribution reduces to exponentiated exponential distribution. For detailed study and properties of Gompertz- Verhulst distribution one may refer to Ahsanullah et al. [26].

It appears from literature that no attention has been paid on the characterization of Gompertz -Verhulst distribution through conditional expectations of dual generalized order statistics and truncation moment.

Throughout the paper, we consider the Case II and then deduce it for Case I.

\section{Characterization Results based on Conditional Expectations}

In this section, we derive the conditional expectations of dual generalized order statistics from the Gompertz Verhulst distribution.

$$
\gamma_{i} \neq \gamma_{j}, \quad i \neq j, \quad i, j=1,2, \ldots, n-1 .
$$

Theorem 2.1: Let $X^{\prime}(r, n, \tilde{m}, k), \quad r=1,2, \ldots \ldots n$ be the $r^{\text {th }}$ dual generalized order statistics from a continuous population with $d f F(x)$ and the $p d f f(x)$. Then for $1 \leq r \leq s \leq n$,

$$
E\left[\xi\left\{X^{\prime}(s, n, \tilde{m}, k)\right\} \mid X^{\prime}(l, n, \tilde{m}, k)=x\right]=\xi(x) a_{s \mid l}+b_{s \mid l}, l=r, r+1
$$


if and only if

$$
F(x)=\left[1-\rho e^{-\lambda x}\right]^{\alpha}, \beta \leq x<\infty
$$

where,

$a_{s \mid r}=\prod_{i=r+1}^{s} \frac{\alpha \gamma_{i}}{1+\alpha \gamma_{i}}, b_{s \mid r}=\frac{1}{\rho}\left[1-a_{s \mid r}\right]$ and $\xi(y)=e^{-\lambda y}$

Proof: We have from (9),

$$
\begin{gathered}
E\left[\xi\left\{X^{\prime}(s, n, \tilde{m}, k)\right\} \mid X^{\prime}(l, n, \tilde{m}, k)=x\right]=\int_{\beta}^{x} \frac{c_{s-1}}{c_{r-1}} \sum_{i=r+1}^{s} a_{i}^{(r)}(s) e^{-\lambda y}\left[\frac{F(y)}{F(x)}\right]^{\gamma_{i}-1} \frac{f(y)}{F(x)} d y \\
=\frac{c_{s-1}}{c_{r-1}} \sum_{i=r+1}^{s} a_{i}^{(r)}(s) \int_{\beta}^{x} e^{-\lambda y}\left[\frac{F(y)}{F(x)}\right]^{\gamma_{i}-1} \frac{f(y)}{F(x)} d y .
\end{gathered}
$$

Let, $\mathrm{U}=\left[\frac{F(y)}{F(x)}\right]=\left[\frac{1-\rho e^{-\lambda y}}{1-\rho e^{-\lambda x}}\right]^{\alpha}$ from (9) in (14)

$$
=\frac{c_{s-1}}{c_{r-1}} \sum_{i=r+1}^{s} a_{i}^{(r)}(s) \frac{1}{\rho} \int_{0}^{1}\left[1-u^{\frac{1}{\alpha}}\left(1-\rho e^{-\lambda x}\right)\right] u^{\gamma_{i}-1} d u .
$$

After simplification we have,

$$
\begin{gathered}
E\left[\xi\left\{X^{\prime}(s, n, \tilde{m}, k)\right\} \mid X^{\prime}(l, n, \tilde{m}, k)=x\right]=\prod_{i=r+1}^{s} \frac{\alpha \gamma_{i}}{1+\alpha \gamma_{i}} e^{-\lambda x}+\frac{1}{\rho}\left[1-\prod_{i=r+1}^{s} \frac{\alpha \gamma_{i}}{1+\alpha \gamma_{i}}\right] \\
E\left[\xi\left\{X^{\prime}(s, n, \tilde{m}, k)\right\} \mid X^{\prime}(l, n, \tilde{m}, k)=x\right]=a_{s \mid r} e^{-\lambda x}+b_{s \mid r} .
\end{gathered}
$$

This proves the necessary part.

To prove the sufficiency part.

Let,

$$
E\left[\xi\left\{X^{\prime}(s, n, \tilde{m}, k)\right\} \mid X^{\prime}(l, n, \tilde{m}, k)=x\right]=g_{s \mid r}(x)
$$

Using the result Khan et al. [16],

$$
\frac{f(x)}{F(x)}=\frac{1}{\gamma_{r+1}}\left[\frac{g_{s \mid r}^{\prime}(x)}{g_{s \mid r+1}(x)-g_{s \mid r}(x)}\right] .
$$

Therefore,

$$
\frac{f(x)}{F(x)}=\frac{\alpha \rho \lambda e^{-\lambda x}}{\left[1-\rho e^{-\lambda x}\right]}
$$

Integrating (16) on both the sides with respect to $x$ between $(\beta, y)$, the sufficiency part is proved.

\section{Remarks}

i) At $\rho=1$, Theorem 2.1 reduces to the characterization result for exponentiated exponential distribution.

ii) Setting $m=0, k=1$ in (12), we obtain the characterization results for Gompertz- Verhulst distribution based on reversed order statistics as,

$$
E\left[\xi\left\{X_{s: n}^{\prime} \mid X_{r: n}^{\prime}=x\right]=a_{s \mid r} e^{-\lambda x}+b_{s \mid r}\right.
$$

where,

$$
a_{s \mid r}=\prod_{i=r+1}^{s} \frac{\alpha(n-i+1)}{1+\alpha(n-i+1)}, b_{s \mid r}=\frac{1}{\rho}\left[1-a_{s \mid r}\right] .
$$

And for order statistics,

$$
E\left[\xi\left(X_{n-s+1: n}\right) \mid X_{n-r+1: n}=x\right]=a_{s \mid r} e^{-\lambda x}+b_{s \mid r}
$$


as $X_{r: n}^{\prime}=X_{n-r+1: n}$ where $X_{r: n}$ is the $r^{t h}$ order statistic.

iii) Letting $m \rightarrow 1$ in the (12), we obtain the characterization results for Gompertz- Verhulst distribution based on lower record values as,

$$
E\left[\xi\left(X_{L(s)} \mid X_{L(r)}\right)=x\right]=a_{s \mid r} e^{-\lambda x}+b_{s \mid r},
$$

where,

$$
a_{s \mid r}=\left(\frac{\alpha k}{1+\alpha k}\right)^{s-r}, b_{s \mid r}=\frac{1}{\rho}\left[1-a_{s \mid r}\right] .
$$

iv) Putting $m=0, k=1, s=n$ and $l=n-1$ in equation (12), we get characterization results for GompertzVerhulst distribution based on left truncated moment of order statistics as follows,

$$
E[\xi(X) \mid X \geq x]=E\left[\xi\left(X_{n: n} \mid X_{n-1: n}=x\right)\right]=\frac{\xi(X)}{2} .
$$

Theorem 2.2: Let $X^{\prime}(r, n, \tilde{m}, k), \quad r=1,2, \ldots \ldots n$ be the $r^{t h}$ dual generalized order statistics from a continuous population with $d f F(x)$ and the $p d f f(x)$. Then for $1 \leq r \leq s \leq t \leq n$,

$$
\begin{gathered}
E\left[\xi\left\{X^{\prime}(s, n, \tilde{m}, k) \mid X^{\prime}(l, n, \tilde{m}, k)=x\right]=a_{t \mid s}\left[E \left[\xi\left\{X^{\prime}(s, n, \tilde{m}, k) \mid X^{\prime}(l, n, \tilde{m}, k)=x\right]+b_{t \mid s}\right.\right.\right. \\
, l=r, r+1
\end{gathered}
$$

if and only if

$$
F(x)=\left[1-\rho e^{-\lambda x}\right]^{\alpha}, \beta \leq x<\infty,
$$

where

$$
\begin{aligned}
& a_{t \mid s}=\prod_{i=s+1}^{t} \frac{\alpha \gamma_{i}}{1+\alpha \gamma_{i}}, b_{s \mid r}=\frac{1}{\rho}\left[1-a_{t \mid s}\right] \text { and } \xi(y)=e^{-\lambda y} \\
& a_{t \mid r}=\prod_{i=r+1}^{t} \frac{\alpha \gamma_{i}}{1+\alpha \gamma_{i}}=a_{t \mid s} a_{s \mid r}, \quad b_{t \mid r}=\frac{1}{\rho}\left[1-a_{t \mid r}\right] .
\end{aligned}
$$

Proof: For the necessary part, in view of Theorem 2.1, we have

$$
\begin{gathered}
E\left[\xi\left\{X^{\prime}(t, n, \tilde{m}, k) \mid X^{\prime}(r, n, \tilde{m}, k)=x\right]=a_{t \mid r} e^{-\lambda x}+b_{t \mid r}\right. \\
E\left[\xi\left\{X^{\prime}(t, n, \tilde{m}, k) \mid X^{\prime}(r, n, \tilde{m}, k)=x\right]=a_{t \mid s} a_{s \mid r}\left[e^{-\lambda x}-\frac{1}{\rho}\right]+\frac{1}{\rho}\right. \\
=a_{t \mid s} a_{s \mid r}\left[e^{-\lambda x}-\frac{1}{\rho}\right]+\frac{1}{\rho} \\
=a_{t \mid s}\left[a_{s \mid r}\left[e^{-\lambda x}-\frac{1}{\rho}\right]+\frac{1}{\rho}\right]-\frac{a_{t \mid s}}{\rho}+\frac{1}{\rho} \\
=a_{t \mid s} E\left[\xi\left\{X^{\prime}(t, n, \tilde{m}, k) \mid X^{\prime}(r, n, \tilde{m}, k)=x\right]+b_{t \mid s}\right. \\
E\left[\xi\left\{X^{\prime}(t, n, \tilde{m}, k) \mid X^{\prime}(r, n, \tilde{m}, k)=x\right]=a_{t \mid s} E\left[\xi\left\{X^{\prime}(t, n, \tilde{m}, k) \mid X^{\prime}(r, n, \tilde{m}, k)=x\right]+b_{t \mid s}\right.\right.
\end{gathered}
$$

Hence the necessary part is proved.

For sufficiency part, we have

$$
\begin{aligned}
& \frac{c_{t-1}}{c_{r-1}} \sum_{i=r+1}^{t} a_{i}^{(r)}(t) \int_{\beta}^{x} e^{-\lambda y}\left[\frac{F(y)}{F(x)}\right]^{\gamma_{i}} \frac{f(y)}{F(y)} d y \\
= & a_{t \mid s} \frac{c_{s-1}}{c_{r-1}} \sum_{i=r+1}^{s} a_{i}^{(r)}(s) \int_{\beta}^{x} e^{-\lambda y}\left[\frac{F(y)}{F(x)}\right]^{\gamma_{i}} \frac{f(y)}{F(y)} d y+b_{t \mid s}
\end{aligned}
$$


Differentiating (19) with respect to $x$ and rearranging we get,

$$
\begin{gathered}
\frac{c_{t-1}}{c_{r-1}} \sum_{i=r+1}^{t} a_{i}^{(r)}(t) e^{-\lambda x}-\frac{c_{t-1}}{c_{r-1}} \sum_{i=r+1}^{t} \gamma_{i} a_{i}^{(r)}(t) \int_{\beta}^{x} e^{-\lambda y}\left[\frac{F(y)}{F(x)}\right]^{\gamma_{i}} \frac{f(y)}{F(y)} d y \\
=a_{t \mid s}\left[\frac{c_{s-1}}{c_{r-1}} \sum_{i=r+1}^{s} a_{i}^{(r)}(s) e^{-\lambda y}-\frac{c_{s-1}}{c_{r-1}} \sum_{i=r+1}^{s} \gamma_{i} a_{i}^{(r)}(s) \int_{\beta}^{x} e^{-\lambda y}\left[\frac{F(y)}{F(x)}\right]^{\gamma_{i}} \frac{f(y)}{F(y)} d y\right] .
\end{gathered}
$$

After noting that

$$
\begin{aligned}
& \sum_{i=r+1}^{s} a_{i}^{(r)}(s)=0, c_{r}=\gamma_{r+1} c_{r-1} \\
& a_{i}^{(r+1)}(t)=\left(\gamma_{r+1}-\gamma_{i}\right) a_{i}^{(r)}(t) .
\end{aligned}
$$

We get,

$$
\begin{aligned}
& \gamma_{r+1} \frac{c_{t-1}}{c_{r-1}} \sum_{i=r+1}^{t} a_{i}^{(r)}(t) \int_{\beta}^{x} e^{-\lambda y}\left[\frac{F(y)}{F(x)}\right]^{\gamma_{i}} \frac{f(y)}{F(y)} d y \\
& -\gamma_{r+1} \frac{c_{t-1}}{c_{r}} \sum_{i=r+2}^{t} \gamma_{i} a_{i}^{(r)}(t) \int_{\beta}^{x} e^{-\lambda y}\left[\frac{F(y)}{F(x)}\right]^{\gamma_{i}} \frac{f(y)}{F(y)} d y \\
& =a_{t \mid s}\left[\gamma_{r+1} \frac{c_{s-1}}{c_{r-1}} \sum_{i=r+1}^{s} a_{i}^{(r)}(s) \int_{\beta}^{x} e^{-\lambda y}\left[\frac{F(y)}{F(x)}\right]^{\gamma_{i}} \frac{f(y)}{F(y)} d y\right] \text {. } \\
& -a_{t \mid s}\left[\gamma_{r+1} \frac{c_{s-1}}{c_{r}} \sum_{i=r+2}^{s} \gamma_{i} a_{i}^{(r)}(s) \int_{\beta}^{x} e^{-\lambda y}\left[\frac{F(y)}{F(x)}\right]^{\gamma_{i}} \frac{f(y)}{F(y)} d y\right] \text {. }
\end{aligned}
$$

That is,

$$
\gamma_{r+1}\left[g_{t \mid r}(x)-g_{t \mid r+1}(x)\right]=a_{t \mid s} \gamma_{r+1}\left[g_{s \mid r}(x)-g_{s \mid r+1}(x)\right]
$$

where,

$$
g_{s \mid r}(x)=\left[E \left[\xi\left\{X^{\prime}(s, n, \tilde{m}, k) \mid X^{\prime}(r, n, \tilde{m}, k)=x\right]\right.\right.
$$

or,

$$
g_{t \mid r}(x)-a_{t \mid s} g_{s \mid r}(x)=g_{t \mid r+1}(x)-a_{t \mid s} g_{s \mid r+1}(x)=\ldots g_{t \mid s}(x)-a_{t \mid s} g_{s \mid s}(x)=b_{t \mid s} .
$$

Noting that, $g_{s \mid s}(x)=e^{-\lambda x}$, we have

$$
g_{t \mid s}(x)=a_{t \mid s} e^{-\lambda x}+b_{t \mid s}
$$

i.e.,

$$
E\left[\xi\left\{X^{\prime}(s, n, \tilde{m}, k) \mid X^{\prime}(r, n, \tilde{m}, k)=x\right]=a_{t \mid s} e^{-\lambda x}+b_{t \mid s} .\right.
$$

Using the result Khan et al. [16],

$$
E\left[\xi\left\{X^{\prime}(s, n, \tilde{m}, k) \mid X^{\prime}(r, n, \tilde{m}, k)=x\right]=g_{t \mid s}(x),\right.
$$

implies,

$$
F(x)=e^{-\int_{\beta}^{x} A(u) d u},
$$

where,

$$
A(u)=\frac{1}{\gamma_{r+1}}\left[\frac{g_{t \mid s}^{\prime}(u)}{g_{t \mid s+1}(u)-g_{t \mid s}(u)}\right],
$$


we get,

$$
F(x)=\left[1-\rho e^{-\lambda x}\right]^{\alpha}, \beta \leq x<\infty .
$$

and hence the Theorem.

\section{Remarks}

v) Putting $s=r$ in (17), Theorem 2.2 reduces to Theorem 2.1 .

vi) At $m=0, k=1$ in Theorem 2.2, we get the result for order statistics,

$$
\begin{gathered}
E\left[\xi\left\{\left(X_{r: n}\right)\right\} \mid X_{t: n}=y\right]=a_{r \mid s} E\left[\xi\left\{\left(X_{s: n}\right)\right\} \mid X_{t: n}=y\right]+b_{r \mid s} \\
a_{r \mid s}=\prod_{j=s}^{s-1} \frac{\alpha j}{1+\alpha j}, b_{s \mid r}=\frac{1}{\rho}\left[1-a_{r \mid s}\right] .
\end{gathered}
$$

vii) It may be seen that when $\gamma_{i} \neq \gamma_{j}$ but $m_{i}=m_{j}=m$.

$$
\begin{gathered}
a_{i}^{(r)}(t)=\frac{1}{(m+1)^{t-r-1}}(-1)^{t-i} \frac{1}{(i-r-1) !(t-1) !} \\
a_{i}(r)=\frac{1}{(m+1)^{r-1}}(-1)^{r-i} \frac{1}{(i-1) !(r-1) !}
\end{gathered}
$$

and consequently (5) will reduces to (2) and (6) to (3).

\section{Characterization Results based on Truncated Moments}

Following theorem contains characterization result for Gompertz- Verhulst distribution based on truncated moments.

Theorem 3.1: Suppose an absolutely continuous (with respect to Lebesgue measure) random variable $X$ has the df $F(x)$ and $p d f f(x)$ for $x \geq 0$ such that $f^{\prime}(x)$ and $E(X \mid X \leq x)$ exist then

$$
E(X \mid X \leq x)=g(x) \eta(x)
$$

where,

if and only if

$$
\eta(x)=\frac{f(x)}{F(x)}, g(x)=\frac{x\left(1-\rho e^{-\lambda x}\right)}{\lambda \alpha \rho e^{-\lambda x}\left(1-\rho e^{-\lambda x}\right)^{\alpha-1}}-\frac{\int_{\beta}^{x}\left(1-\rho e^{-\lambda x}\right)^{\alpha} d u}{\lambda \alpha \rho e^{-\lambda x}\left(1-\rho e^{-\lambda x}\right)^{\alpha-1}}
$$

$$
f(x)=\lambda \alpha \rho e^{-\lambda x}\left(1-\rho e^{-\lambda x}\right)^{\alpha-1}, \beta \leq x<\infty .
$$

Proof: We have,

$$
E(X \mid X \leq x)=\frac{1}{F(x)} \int_{\beta}^{x} u f(u) d u=\frac{\lambda \alpha \rho}{F(x)} \int_{\beta}^{x} u e^{-\lambda u}\left(1-\rho e^{-\lambda u}\right)^{\alpha-1} d u .
$$

Integrating (21) by parts treating $e^{-\lambda u}\left(1-\rho e^{-\lambda u}\right)^{\alpha-1}$ for integration and rest for the integrand for differentiation, we get,

$$
E(X \mid X \leq x)=\frac{1}{F(x)}\left\{x\left(1-\rho e^{-\lambda x}\right)-\int_{\beta}^{x}\left(1-\rho e^{-\lambda x}\right)^{\alpha} d u\right\} .
$$

After multiplying and dividing by $f(x)$ in (22), we have the result given (20)

$$
E(X \mid X \leq x)=\frac{x\left(1-\rho e^{-\lambda x}\right)}{\lambda \alpha \rho e^{-\lambda x}\left(1-\rho e^{-\lambda x}\right)^{\alpha-1}}-\frac{\int_{\beta}^{x}\left(1-\rho e^{-\lambda x}\right)^{\alpha} d u}{\lambda \alpha \rho e^{-\lambda x}\left(1-\rho e^{-\lambda x}\right)^{\alpha-1}} \frac{f(x)}{F(x)}
$$




$$
E(X \mid X \leq x)=g(x) \eta(x) .
$$

To prove the sufficiency part, we have from (20)

$$
\frac{1}{F(x)} \int_{\beta}^{x} u f(u) d u=\frac{g(x) f(x)}{F(x)} \text { or } \int_{\beta}^{x} u f(u) d u=g(x) f(x) .
$$

Differentiating (23) on both the sides with respect to $x$, we have

$$
x f(x)=g^{\prime}(x) f(x)+g(x) f^{\prime}(x) .
$$

Therefore,

$$
\begin{array}{lr}
\frac{f^{\prime}(x)}{f(x)}=\frac{x-g^{\prime}(x)}{g(x)} & \text { Ahsanullah et al. [27] } \\
\frac{f^{\prime}(x)}{f(x)}=\frac{x-g^{\prime}(x)}{g(x)}=\left[-\lambda+\frac{(\alpha-1) \lambda \rho e^{-\lambda x}}{\left(1-\rho e^{-\lambda x}\right)}\right]
\end{array}
$$

where,

$$
g^{\prime}(x)=x+g(x)\left[-\lambda+\frac{(\alpha-1) \lambda \rho e^{-\lambda x}}{\left(1-\rho e^{-\lambda x}\right)}\right] .
$$

Integrating both sides in (24) with respect to $x$, we get,

$$
f(x)=c e^{-\lambda x}\left(1-\rho e^{-\lambda x}\right)^{\alpha-1} .
$$

Now, using the condition, $\int_{-\infty}^{\infty} f(x) d x=1$

$$
\begin{gathered}
\int_{\beta}^{x} c e^{-\lambda x}\left(1-\rho e^{-\lambda x}\right)^{\alpha-1} d x=1 \\
\frac{1}{c}=\int_{\beta}^{x} e^{-\lambda x}\left(1-\rho e^{-\lambda x}\right)^{\alpha-1} d x \\
\frac{1}{c}=\frac{1}{\alpha \rho \lambda} .
\end{gathered}
$$

This proves that,

$$
f(x)=\lambda \alpha \rho e^{-\lambda x}\left(1-\rho e^{-\lambda x}\right)^{\alpha-1}, \beta \leq x<\infty .
$$

\section{Remark}

viii) Putting $\rho=1$, in Theorem 3.1 we get the result for exponentiated exponential distribution as obtained by Ahsanullah et al. [27].

\section{Conclusion}

Characterization of Gomertz-Verhulst distribution has been considered by conditional expectations based on non-adjacent dual generalized order statistics and truncation moment. These characterization results are useful in the field of ordered random variables. Findings of this paper will be useful for researchers in the fields of applied sciences, population studies and further enhancement of research in the field of distribution theory and its applications. 


\section{Acknowledgement}

The author is grateful to the Coordinating Editor and learned referee for providing valuable comments which led to an improvement in the present manuscript. This research was supported by the Deanship of Scientific Research [Research Project No. (06/40)], Islamic University of Madinah, KSA.

\section{REFERENCES}

1. M. Q. Shahbaz, M. Ahsanullah, S. H. Shahbaz and B. M. Al-Zahrani, Ordered Random Variables: Theory and Applications, Atlantis Press, 2016.

2. U. Kamps, A concept of Generalized Order Statistics, Teubner Stuttgart, 1995.

3. M. Burkschat, E. Cramer and U. Kamps, Dual generalized order statistics, Metron, vol.61, pp.13-26, 2003.

4. A. M.Kagan, Yu. V. Linnik and C. R. Rao, Characterization Problems in Mathematical Statistics, John Wiley \& Sons, New York (English Translation, translated from the Russian text by B. Ramachandran), 1973.

5. I. I. Kotlarski, On a characterization of some probability distributions by conditional expectations, Sankhya, vol.34, no. 4 pp. 461-466, 1972.

6. S. Talwalker, A note on characterization by conditional expectation, Metrika, vol.24, pp.129-136, 1977.

7. J. Galambos and S. Kotz, Characterization of probability distributions. A unified approach with an emphasis of exponential and related models, Lecture Notes in Mathematics, 675, Springer, Berlin, Germany, 1978.

8. R. C. Gupta and M. Ahsanullah, Some characterization results based on the conditional expectation of a function of non- adjacent order statistic (Record value), Ann. Inst. Statist. Math., vol.56, no.4 pp.721-732, 2004.

9. P. Zora, R. M. Ruiz and J. Marin, A characterization based on conditional expectation, Comm. Statist. Theory Methods, vol.16, pp. 3127-3135, 1990.

10. R. U. Khan, M. A. Khan and M. A. R. Khan, Relations for moments of generalized record values from additive Weibull distribution and associated inference, Stat., Optim. Inf. Comput., vol. 5, pp.127-136, 2017.

11. N. Gupta and Z. Anwar, Relations for single and product moments of odd generalized exponential- Pareto distribution and characterization, Stat., Optim. Inf. Comput., vol. 7, pp.160-170, 2019.

12. M. Ahsanullah, A characterization of the uniform distribution by dual generalized order statistics, Comm. Stat. Theory Methods, vol.33, pp.2921-2928, 2004.

13. M. Ahsanullah, On lower generalized order statistics and a characterization of the power function distribution, Stat. Methods, vol.7, no.1 pp.16-28, 2005.

14. A. K. Mbah and M. Ahsanullah, Some characterization of the power function distribution based on lower generalized order statistics, Pak. J. Stat., vol.23, pp.139-146, 2007.

15. A. H. Khan, M. Faizan and Z. Haque, Characterization of probability distributions by conditional variance of generalized order statistics and dual generalized order statistics, J. Statist. Theory Appl., vol.9, pp.375-385, 2010.

16. A. H. Khan, Z. Anwar and S. Chishti, Characterization of continuous distributions through conditional expectation of functions of dual generalized order statistics, Pakistan. J. Stat., vol. 26, pp.615-628, 2010.

17. M. Faizan and M. I. Khan, Characterization of some probability distributions by conditional expectations of dual generalized order statistics, J. Stat. Sci., vol.3, no.2 pp.143-150, 2011.

18. M. Tavanagar, Power function distribution characterized by dual generalized order statistics, J. Iran. Stat. Soci., vol.10, no.1 pp.13-27, 2011.

19. M. I. Khan and M. Faizan, Some characterization results based on conditional expectation of function of dual generalized order statistics, Pak. J. Stat. Oper. Res., vol.8, no.4 pp.789-799, 2012.

20. R. U. Khan and M. A. Khan, Dual generalized order statistics from family of J-shaped distribution and characterization, Journal of King Saud University- Science, vol. 27, pp. 285-291, 2015.

21. M. I. Khan and M. A. R. Khan, Characterization of Burr-Type X distribution based on conditional expectations of dual generalized order statistics, Int. J. Comp. Theo. Stat., vol. 4, no.1 pp.37-43, 2017.

22. B. Gompertz, On the nature of the function expressive of the law of human mortality and on a new mode of determining the value of life contingencies, Philosophical Transactions of the Royal Society London, vol.115, pp. 513-585, 1825.

23. P. F. Verhulst, Recherches mathematiques sur la loi-d-accroissement de la population. Nouvelles Memoires de 1 Academie Royale des Sciences et Belles- Lettres de Bruxellesi. (i,e, Memories, series 2), vol.18, pp. 38, 1845.

24. P. F.Verhulst, Deuxieme memoire sur la loi-d-accroissement de la population. Memoires de 1 Academie Royale des Science, des Lettres et des Beaux- Arts de Belgique, Series 2, vol. 20, pp. 32, 1847.

25. P. F.Verhulst, Notice sur la loi la population suit dans son accroissement. Correspondence mathematique et physique, publiee L. A. J. Quetelet, vol.10, pp.113-121, 1838.

26. M. Ahsanullah, M. Shakil, and B. M. Golam Kibria, A note on a characterization of Gompertz- Verhulst distribution, J. Statist. Theory Appl., vol.13, no.1 pp.17-26, 2014.

27. M. Ahsanullah, M. Shakil and B. M. Golam Kibria, Characterization of continuous distributions by truncated moment, J. Modern Appl. Satist. Methods, vol.15, no.1 pp.316-331, 2016. 